\title{
Impact of individualized care on readmissions after a hospitalization for acute exacerbation of COPD
}

\author{
This article was published in the following Dove Press journal: \\ International Journal of COPD \\ 5 January 2016 \\ Number of times this article has been viewed
}

\author{
Simon L Adamson' \\ Jane Burns ${ }^{1,2}$ \\ Pat G Camp ${ }^{1,2}$ \\ Don D Sin ${ }^{1,3}$ \\ Stephan F van Eeden ${ }^{1,3}$ \\ 'The Centre for Heart Lung \\ Innovation, St Paul's Hospital, \\ University of British Columbia, \\ ${ }^{2}$ Department of Physical Therapy, \\ ${ }^{3}$ Division of Respirology, Department \\ of Medicine, University of British \\ Columbia, Vancouver, BC, Canada
}

Correspondence: Stephan F van Eeden The Centre for Heart Lung Innovation, St Paul's Hospital, University of British Columbia, I08I Burrard Street,

Vancouver, BC V6ZIY6, Canada

Tel +l 6048068346

Fax + I 604806835 I

Email stephan.vaneeden@hli.ubc.ca
Background: Acute exacerbations of chronic obstructive pulmonary disease (AECOPD) increase COPD morbidity and mortality and impose a great burden on health care systems. Early readmission following a hospitalization for AECOPD remains an important clinical problem. We examined how individualized comprehensive care influences readmissions following an index hospital admission for AECOPD.

Methods: We retrospectively reviewed data of patients admitted for AECOPD to two inner-city teaching hospitals to determine the impact of a comprehensive and individualized care management strategy on readmissions for AECOPD. The control group consisted of 271 patients whose index AECOPD occurred the year before the comprehensive program, and the experimental group consisted of 191 patients who received the comprehensive care. The primary outcome measure was the total number of readmissions in 30- and 90-day postindex hospitalizations. Secondary outcome measures included the length of time between the index admission and first readmission and all-cause mortality.

Results: The two groups were similar in terms of age, sex, forced expiratory volume in 1 second, body mass index (BMI), pack-years, and the number and types of comorbidities. Comprehensive care significantly reduced 90 -day readmission rates in females $(P=0.0205$, corrected for age, BMI, number of comorbidities, substance abuse, and mental illness) but not in males or in the whole group $(P>0.05)$. The average times between index admission and first readmission were not different between the two groups. Post hoc multivariate analysis showed that substance abuse $(P<0.01)$ increased 30- and 90-day readmissions (corrected for age, sex, BMI, number of comorbidities, and mental illness). The 90-day all-cause in-hospital mortality rates were significantly less in the care package group $(2.67 \%$ versus $7.97 \%, P=0.0268)$.

Conclusion: Comprehensive individualized care for subjects admitted to hospital for AECOPD did not reduce 30 - and 90-day readmission rates but did reduce 90 -day total mortality. Interestingly, it reduced 90-day readmission rate in females. We speculate that an individualized care package could impact COPD morbidity and mortality after an acute exacerbation.

Keywords: readmissions, care package, substance abuse, female, mortality

\section{Introduction}

Acute exacerbations of chronic obstructive pulmonary disease (AECOPD) are characterized by a sudden worsening of patients' respiratory symptoms such as shortness of breath, cough, and sputum production ${ }^{1}$ and are defining events, which are associated with increased COPD morbidity and mortality. ${ }^{2,3}$ These events also decrease quality of life, ${ }^{4}$ accelerate the lung function decline, ${ }^{5}$ and are associated with significant health care costs. ${ }^{6}$ Patients with COPD experience the greatest number of readmissions to acute hospital care and the emergency room (ER) visits of any patient group, with more than half of these readmissions due to COPD. ${ }^{7}$ Because of this, decreasing acute exacerbations and reducing readmissions remain the primary goals of COPD management. 
Earlier research has investigated chronic disease management strategies to address the significant burden associated with AECOPD. Most of these studies have been in the form of targeted care programs focused on a combination of patient education, self-management, and outreach health care services. ${ }^{8}$ While some of these programs have improved exacerbation and readmission frequency, ${ }^{9-11}$ others have shown no benefit. ${ }^{12-14}$ Furthermore, these care programs are inconsistent in their effect on patient mortality. This heterogeneity likely stems from the variability of care program components, study designs, and patient populations. In addition, there is an evidence to suggest that certain subpopulations respond differently to care programs than others, ${ }^{10}$ indicating that wholepopulation studies overlooked important subtleties in the COPD management. This suggests that targeted individualized care programs may be more impactful reducing morbidity and mortality following an acute exacerbation of COPD.

This study was designed to determine the effects of a "needbased" individualized care package (CP) delivered to an innercity population of patients hospitalized for AECOPD. The CP was developed to target the sensitive time period (2-4 weeks) immediately following a hospitalization due to AECOPD. ${ }^{2}$ The primary outcome measured was readmission rates at 30 and 90 days and the secondary outcomes investigated were the length of time until first readmission and mortality rates.

\section{Methods}

\section{Patients}

The patients were selected for inclusion into the study if they had had a hospitalization with a primary diagnosis of AECOPD at two inner-city hospitals, St Paul's and Mount St Joseph's Hospitals, between March 1, 2012 and March 1, 2014. The patients were excluded if they lived outside the normal service area of these two hospitals. The CP was fully implemented on March 1, 2013. Therefore, the pre-CP group consisted of patients whose admission occurred between March 1, 2012 and March 1, 2013, before the CP has been enacted. The CP group consisted of patients whose admission occurred between March 1, 2013 and March 1,2014, during the full implementation of the CP.

\section{COPD management}

\section{Standard care}

In-hospital management of AECOPD in all subjects during both periods were standardized using a hospital-wide protocol for AECOPD based on ATS/ERS guidelines. ${ }^{15}$ The day-to-day management of patients was done under the guidance of the admitting physician (either general internist or respirologist).

\section{Care package}

A COPD Outreach Team was assembled to implement and deliver the individualized CP. The team consisted of a nurse practitioner, a respiratory therapist, and a physiotherapist. The patients admitted to hospital due to AECOPD were initially approached by a member of the Outreach Team and enrolled into the program. The CP was divided into four main stages: i) initial contact; ii) postdischarge phone-call/contact; iii) home visit; and iv) continued care. The specific interventions employed at each of these stages are outlined in Figure 1.

The Outreach Team functioned as a cohesive unit with largely overlapping responsibilities of patient care. The multidisciplinary nature of the team also allowed each member to contribute their respective expertise to certain interventions. For example, the physiotherapist was the best able to address fall prevention and encourage mobility, the respiratory therapist to assess breathing and review breathing techniques, and the nurse to provide disease education and perform medication reconciliation. Every home visit was attended by at least two of the three-team members. Not all of the interventions listed in Figure 1 were performed for every patient, and the relative emphasis placed on each was different for every patient. The individualized management plan for each patient was determined based on comprehensive clinical and needs assessment.

Overall, the goal of the program was to provide targeted and individualized disease management by: i) facilitating a seamless transition of the patient back the community; ii) providing disease-specific education and self-management training; iii) identifying and addressing individual risk factors; and iv) mobilizing appropriate community resources and coordinating care providers.

\section{Data collection}

Patient data were retrospectively collected using patient charts and hospital's electronic databases. To ensure that readmissions to other hospitals or deaths were not missed, a provincial-wide electronic database "CareConnect Electronic Health Record" was used. This application collects and centralizes patient demographics, care information, and clinical documents. Ethics approval for this study was obtained from the University of British Columbia and Providence Health Care Research Institute Research Ethics Board. The authors' institutional review board is a combined committee of the University of British Columbia and the St Paul's Hospital, Providence Health Care Research Institute. This board has given consent to use this retrospective data without informed consent because informed consent by all participants was not possible. 


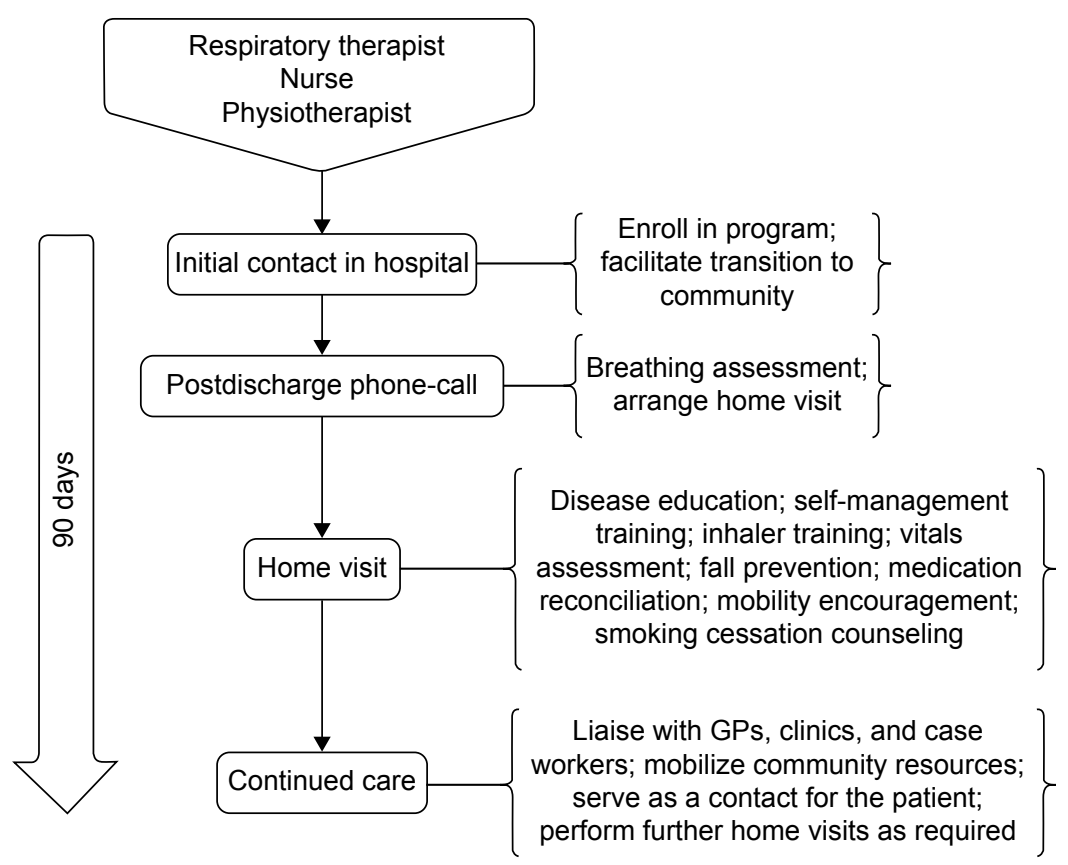

Figure I Components and organization of the care package delivered by the COPD Outreach Team to patients after a hospitalization due to AECOPD. Abbreviations: AECOPD, acute exacerbations of chronic obstructive pulmonary disease; GPs, general practitioners.

\section{Statistical analysis}

Statistical tests used to assess baseline patient characteristics were chosen depending on the number of groups being compared, whether the variable was continuous or proportional, and whether the data was normally distributed or not. For analysis of two groups, the Student's $t$-test and Fisher's exact test were used. For comparison between four groups, the analysis of variance test with a post hoc Tukey test and the chi-square test was used.

Length of time until first readmission was evaluated using the Student's $t$-test. All-cause mortality was assessed through the Kaplan-Meier estimate, using the log-rank test to determine significance. All tests listed earlier were performed using Prism 5 for Windows (GraphPad Software, Inc., San Diego, CA, USA).

The independent effects of the CP on all-cause total, ER, and hospital readmission rates at 30 and 90 days were assessed using predefined multivariant linear regressions. A priori determined covariates included age, sex, number of comorbidities, index admission length of stay, substance abuse (SA), and mental illness. Age, sex, number of comorbidities, and index admission length of stay were included because these variables are known to influence readmissions, ${ }^{16,17}$ while SA and mental illness were included to reflect the nature of the population being studied (ie, high prevalence of SA and mental illness). Forced expiratory volume in 1 second $\left(\mathrm{FEV}_{1}\right)$, body mass index, and pack-years of smoking history were not included because data was not available for all patients and power would have been significantly reduced. Similar regressions were performed to assess the effects of SA and sex on readmissions. These regressions were performed using R-Studio for Mac (RStudio, Inc., Boston, MA, USA). All tests employed in this study were two-tailed and $P$-values $<0.05$ were considered significant.

\section{Results}

\section{Baseline patient characteristics}

A total of 462 patients fulfilled the eligibility criteria and were included in the study. Of these, 271 were part of the control pre-CP group and 191 received the CP. There were no significant differences in baseline demographic characteristics between the two groups (Table 1). Due to the innercity nature of the study population, a substantial portion of the study subjects had a history of SA. Because a history of SA was associated with an increased readmission rate, the patients were stratified as SA if they had a past or current history of SA (recreational drugs and alcohol) or non-SA (NSA) if they had no such history (Table 2). The patients with SA were significantly younger than the patients with NSA with slightly more comorbidities.

\section{Readmissions}

The all-cause readmission rates for patients in the pre-CP and $\mathrm{CP}$ groups are shown in Figure 2. Multivariate linear 
Table I Demographic data of subject groups with AECOPD

\begin{tabular}{|c|c|c|c|}
\hline Variable & Pre-CP $(n=27 I)$ & $C P(n=191)$ & $P$-value \\
\hline Age (years) & $70.8(13.8)$ & $70.2(12.4)$ & 0.6134 \\
\hline Male (\%) & 64.6 & 58.6 & 0.2064 \\
\hline $\mathrm{BMI}\left(\mathrm{kg} / \mathrm{m}^{2}\right)$ & $26.52(7.65)$ & $26.11(7.44)$ & 0.6313 \\
\hline FEV $(\%$ predicted $)$ & $45.65(20.17)$ & $47.85(17.09)$ & 0.3034 \\
\hline Number of comorbidities & $3.7(1.98)$ & $3.8(2.05)$ & 0.7397 \\
\hline Substance abuse (\%) & 18.5 & 17.3 & 0.8061 \\
\hline Mental illness (\%) & 29.9 & 30.8 & 0.8376 \\
\hline Smoking (pack-years) & $39.53(36.16)$ & $42.70(31.45)$ & 0.4087 \\
\hline Index admission LOS (days) & 7.531 (7.30I) & $7.131(7.613)$ & 0.5687 \\
\hline
\end{tabular}

Note: Data are presented as mean (standard deviation) or \% of column totals.

Abbreviations: AECOPD, acute exacerbations of chronic obstructive pulmonary disease; CP, care package; BMI, body mass index; FEV , forced expiratory volume in I second; LOS, length of stay.

regression showed no significant differences in total, ER, or acute care readmissions at 30- or 90-day postindex admissions between the groups. The average time to first readmission was $\sim 30$ days and was not significantly different between groups (Figure 3).

The patients with comorbid SA were found to have significantly greater readmission rates than those without (Figure 4A) both at the 30- and 90-day periods. There was a trend $(P<0.08)$ toward reduction in 90 -day readmission rates in the NSA group but not the SA group (Figure 4B and C).

Male patients were found to have significantly more readmissions to acute care within 30 days than female patients (Figure 5A). Furthermore, the CP was found to significantly decrease 90-day total and 90-day ER readmissions in female patients (Figure 5B), but not male patients (Figure 5C). Summary of the results of the adjusted and unadjusted regressions can be found in Tables S1-S5.

\section{Mortality}

All-cause mortality was compared between the pre-CP and $\mathrm{CP}$ patient groups over 90-day postindex admissions. The pre-CP group was found to have significantly increased allcause mortality compared with the $\mathrm{CP}$ group with a hazard ratio equal to 2.455 (95\% confidence interval: 1.109-5.436; Figure 6). Using the absolute reduction in risk of death at 90 days, 21 patients would have to be treated to prevent one death (95\% confidence interval: $12-110)$.

\section{Discussion}

The primary goal of this study was to determine the impact of an individualized CP on early readmission rates following a hospital admission due to AECOPD. Our results showed that this type of intervention did not alter readmission rates of subjects with moderate-to-severe COPD. In the subgroup analysis, we found that 90-day readmissions were reduced in female patients who received individualized $\mathrm{CP}$, suggesting females were more sensitive to our CP. Because of the proximity of these hospitals to a neighborhood with high rates of poverty, $18 \%$ of subjects had either earlier or current SA problems and our results showed that these patients had increased readmissions both at 30 and 90 days. Interestingly, all-cause mortality at 90 days was reduced in the $\mathrm{CP}$ group. Together, these findings suggest that an individualized CP could impact COPD morbidity and mortality after an acute exacerbation in certain subpopulations.

Table 2 Demographic data of substance abuse (SA) and non-SA (NSA) groups

\begin{tabular}{|c|c|c|c|c|c|}
\hline Variable & SA pre-CP $(n=50)$ & SA CP $(n=33)$ & NSA pre-CP $(n=22 I)$ & NSA CP $(n=158)$ & $P$-value \\
\hline Age (years) & $60.05(12.44)$ & $58.34(8.319)$ & 73.28 (12.09) & 72.71 (11.67) & $<0.000 I^{*}$ \\
\hline Male (\%) & 66.00 & 60.60 & 64.25 & 58.23 & 0.6157 \\
\hline $\operatorname{BMI}\left(\mathrm{kg} / \mathrm{m}^{2}\right)$ & $23.75(6.30)$ & $25.23(9.04)$ & $27.13(7.80)$ & $26.25(7.17)$ & 0.1208 \\
\hline FEV (\% predicted) & 41.31 (16.91) & $52.60(19.67)$ & 46.69 (20.79) & $47.03(16.56)$ & 0.1800 \\
\hline Number of comorbidities & 4.26 & 4.42 & 3.62 & 3.67 & $0.0430 *$ \\
\hline Mental illness (\%) & 32.0 & 33.3 & 29.4 & 30.4 & 0.9609 \\
\hline Smoking (pack-years) & $40.14(22.29)$ & $50.03(28.53)$ & $39.39(38.70)$ & $41.59(31.83)$ & 0.6506 \\
\hline Index admission LOS (days) & $6.76(6.413)$ & $5.49(4.273)$ & $7.71(7.490)$ & $7.48(8.107)$ & 0.4009 \\
\hline
\end{tabular}

Notes: Data are presented as mean (standard deviation) or $\%$ of column totals. $* P<0.05$ difference between the SA and NSA groups.

Abbreviations: $\mathrm{CP}$, care package; $\mathrm{BMI}$, body mass index; $\mathrm{FEV}_{1}$, forced expiratory volume in I second; LOS, length of stay. 


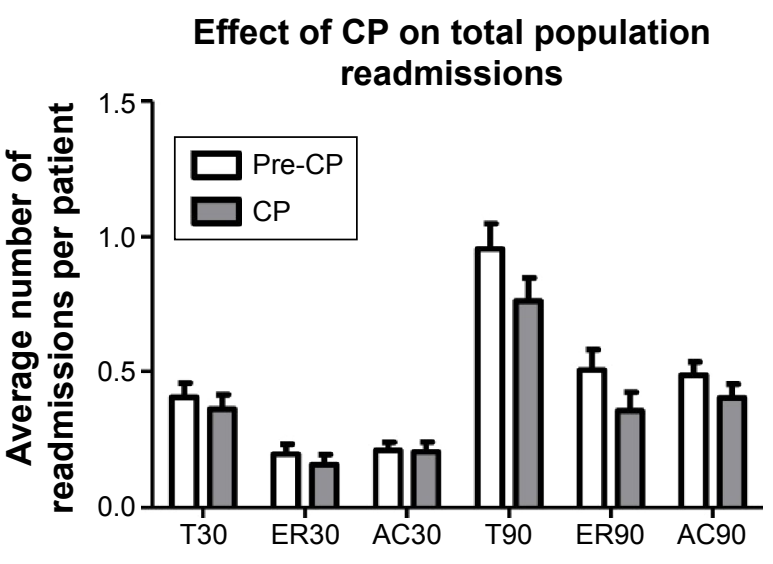

Figure 2 Thirty- and 90-day total, ER, and acute care readmissions in the pre-CP and $\mathrm{CP}$ groups.

Notes: Multivariate linear regression was used to determine significance; adjusted for covariates (age, sex, number of comorbidities, substance abuse, mental illness, and index admission length of stay).

Abbreviations: ER, emergency room; T30, total readmissions in 30 days; ER30, emergency room readmissions in 30 days; AC30, acute care readmissions in 30 days T90, total readmissions in 90 days; ER90, emergency room readmissions in 90 days; AC90, acute care readmissions in 90 days; $\mathrm{CP}$, care package.

AECOPD are defining events in the progression of COPD impacting both morbidity and mortality. ${ }^{2,3,18}$ Whether interventions such as specific "care packages" modify morbidity and mortality, in particular readmissions, are still controversial due to variable success. ${ }^{9-14,19}$ Part of the difficulty of trying to compare studies is the heterogeneity of the interventions enacted, although certain foundational aspects of these CPs, such as disease education and selfmanagement training, are relatively universal. In addition, patient populations, follow-up lengths, and outcomes measured differ greatly between studies. Several systematic reviews ${ }^{8,19,20}$ have highlighted this controversy and it is still uncertain why some CPs work while others do not, whether it is the level of intervention that plays a role ${ }^{8}$ or the patient population/COPD phenotype. However, these reviews all

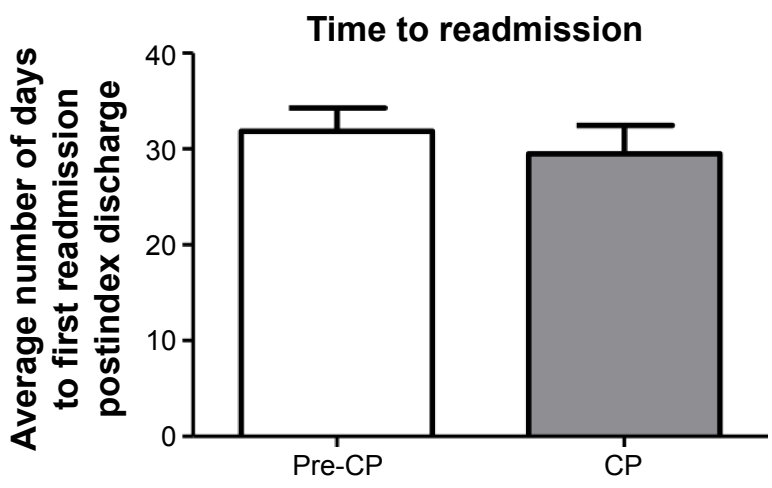

Figure 3 Average time to readmission in pre-CP and CP groups. Notes: Two-tailed Student's $t$-test used to determine significance; $P=0.5430$. Abbreviation: $\mathrm{CP}$, care package. recognize the potential value of these CPs and the need for further study to determine how COPD management can be better optimized.

We looked to determine whether a CP individualized to each subject's circumstance could reduce early readmission rates in subjects admitted to the hospital for an AECOPD. Our study showed that our CP did not alter COPD readmissions in the whole group, a result similar to a recent randomized control trial by Jennings et al. ${ }^{14}$ Our patient population was similar to those in the randomized trail in terms of demographics and disease severity, although the interventions differed significantly. The study by Jennings et al ${ }^{14}$ used a fixed, standardized intervention on discharge with one phone call 48 hours following discharge. In contrast to this management plan and many of the other interventions previously investigated in the literature, our $\mathrm{CP}$ was purposely designed to be flexible so that it could be individualized to each patient to address the unique medical and social needs. Which interventions were included and emphasized for each patient was up to the discretion of the Outreach Team and depended objective clinical parameters and subjective assessments of situational factors as well as the willingness and abilities of the particular patient. Invaluable to the subjective assessments and unique to our $\mathrm{CP}$ was the inclusion of one or more home visits by two or more health professionals. Furthermore, central to our study was the integration of patients with community resources and continued follow-up by phone calls or home visits.

Our hospital serves a large inner-city population and a significant proportion $(-30 \%)$ of patients are residents of the Downtown Eastside, a marginalized population where crime, unemployment, homelessness, poverty, poor education, SA, and chronic infections are prevalent. ${ }^{21,22}$ These subjects frequently have no fixed address or phone number and have limited social support. As these factors have been associated with increased barriers to care, ${ }^{23}$ decreased ability to self-manage disease, ${ }^{24}$ and poor adherence to management regimens, ${ }^{25}$ we suspect that these factors played a role in why we were unable to show a significant impact of our $\mathrm{CP}$ in the whole group of subjects. To further support this notion, $\sim 20 \%$ of the population served by the Outreach Team had significant SA issues, many subjects with polysubstance abuse, including significant intravenous drug use. A history of current or earlier SA was the strongest risk factor for readmission (Figure 4A) of all the factors included in the multivariate analysis. There was a trend toward a reduction in 90-day readmissions (Figure 4C) in the NSA group, but the CP did not impact the readmissions in the SA group, suggesting this 

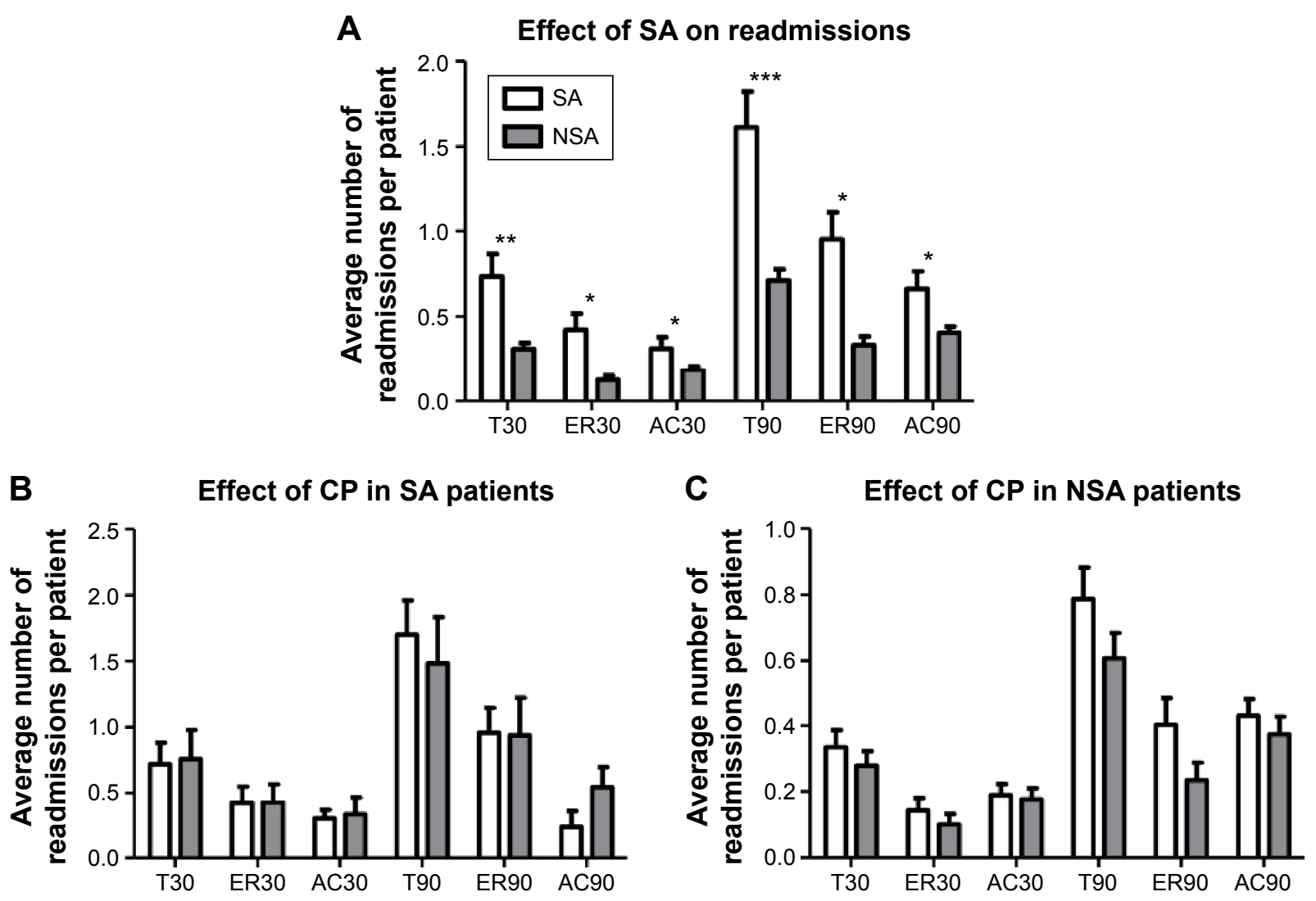

Pre-CP $\square \mathrm{CP}$

Figure 4 (A) Effect of SA on readmissions. Multivariate linear regression used to determine significance; adjusted for covariates (age, sex, number of comorbidities, mental illness, care package, and index admission length of stay). (B and C) Effects of CP in SA and NSA patients, respectively. Multivariate linear regression used to determine significance in each; adjusted for covariates (age, sex, number of comorbidities, mental illness, and index admission length of stay).

Notes: $* P<0.05, * * P<0.01$, *** $P<0.001$.

Abbreviations: SA, substance abuse; NSA, nonsubstance abuse; CP, care package; T30, total readmissions in 30 days; ER30, emergency room readmissions in 30 days; $A C 30$, acute care readmissions in 30 days; T90, total readmissions in 90 days; ER90, emergency room readmissions in 90 days; AC 90 , acute care readmissions in 90 days.

group of subjects needs a different interventional strategy to improve readmissions.

Interestingly, the average $\mathrm{FEV}_{1}$ in the $\mathrm{SA}$ group was similar to that in the NSA group even though the patients were on average $\sim 14$ years younger, suggesting more severe COPD at a younger age in the SA group. The explanation for this is most likely multifactorial and may include more complex lung disease such as associated bronchiectasis, interstitial lung disease, or the direct effects of smoking drugs (such as cocaine). These patients could also have an accelerated progression of their lung disease, as has been documented in such subjects with HIV. ${ }^{26}$ This needs further investigation.

Subgroup analysis showed that readmissions at 90 days in female patients were reduced by the $\mathrm{CP}$ intervention, suggesting that females are more sensitive to this mode of intervention than males. This effect was predominantly driven by a reduction in ER visits (Figure 5B). Disease and self-management education may have helped patients recognize and treat less severe exacerbations earlier and more effectively, thereby decreasing the need to visit the ER. The CP may have also improved day-to-day COPD management and reduced the frequency of less severe exacerbations. As in most large COPD studies, males predominated our study and we suspect that this could be the reason why our total population results were negative. Female sex as an independent factor was not associated with more frequent readmissions while male sex was (Figure 5A), further strengthening our finding that females were more sensitive to the $\mathrm{CP}$.

To our knowledge, only Moullec et a ${ }^{10}$ have previously assessed sex-related differences in response to a care program in patients with COPD. They too found that women responded significantly better than men. A large body of research has demonstrated significant differences in COPD manifestation and experience between men and women. On radiological examination, men and women present with different lung pathology phenotypes, with men showing relatively greater emphysema and less airway involvement. ${ }^{27,28}$ Women also have a different comorbid profile compared with $\operatorname{men}^{29}$ and 

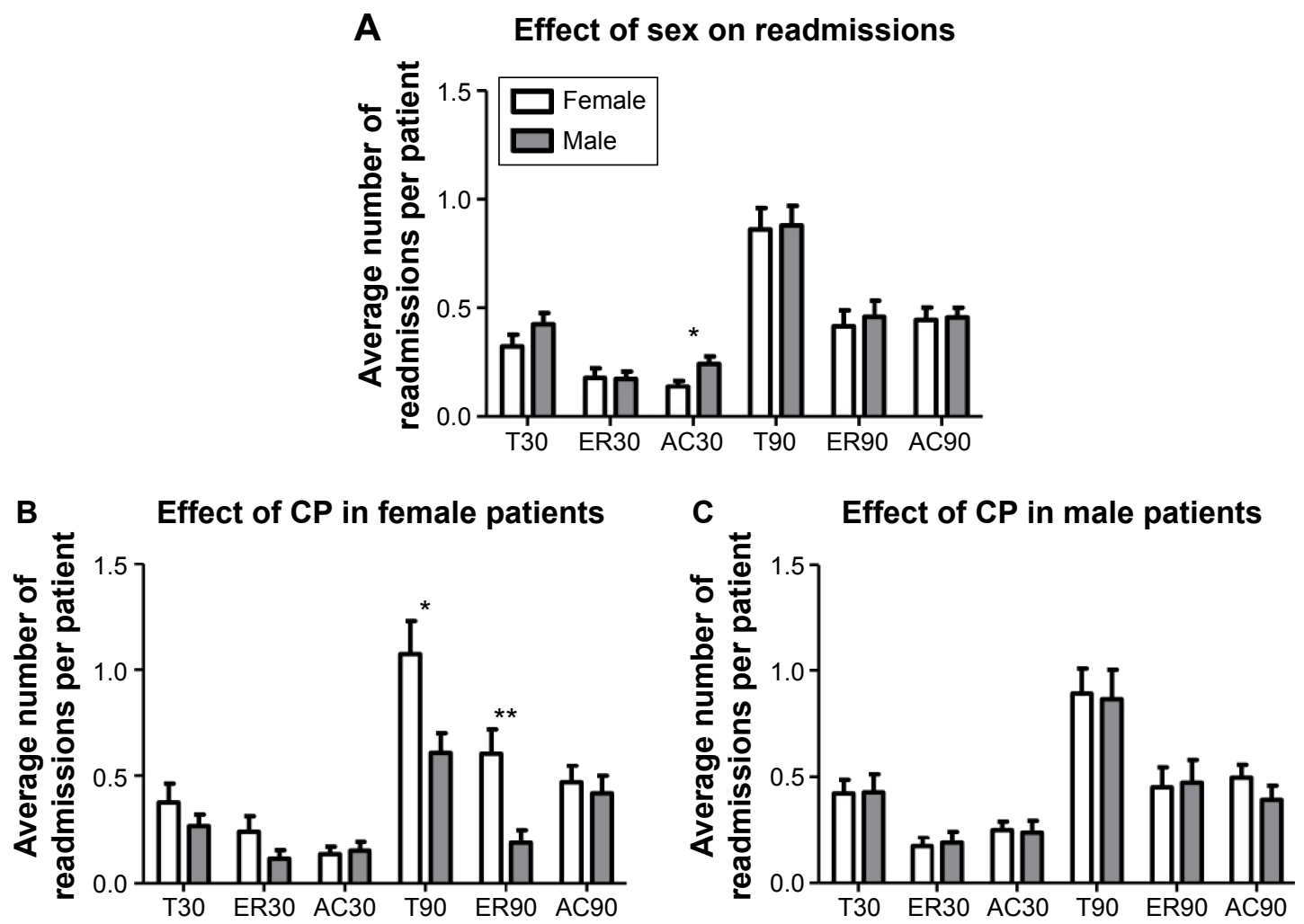

Pre-CP $\square \mathrm{CP}$

Figure 5 (A) Effect of patient sex on readmissions. Multivariate linear regression used to determine significance; adjusted for covariates (age, number of comorbidities, SA, mental illness, index admission length of stay). (B and C) Effect of CP in female and male patients, respectively. Significant improvements found only in female T90 and ER90 readmissions. Multivariate linear regression used to determine significance; adjusted for covariates (age, number of comorbidities, SA, mental illness, index admission length of stay).

Notes: $* P<0.05, * * P<0.01$

Abbreviations: CP, care package; T30, total readmissions in 30 days; ER30, emergency room readmissions in 30 days; AC30, acute care readmissions in 30 days; T90, total readmissions in 90 days; ER90, emergency room readmissions in 90 days; AC 90 , acute care readmissions in 90 days.

experience COPD differently, reporting greater dyspnea, functional limitation, and psychological distress. ${ }^{30,31}$ These differences may also play a role in the sex-difference in response to intervention.

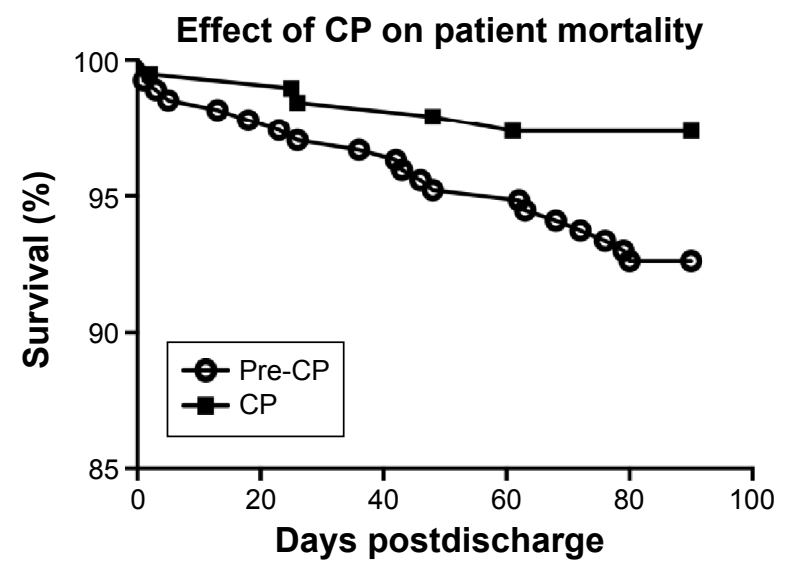

Figure 6 Effect of CP on all-cause in-hospital patient mortality. Notes: Kaplan-Meier estimate was used with a log-rank test to determine significance; $P=0.0286$. Hazard ratio $=2.455$ (95\% confidence interval: I. I09-5.436). Abbreviation: $\mathrm{CP}$, care package.
Somewhat unexpectedly, we found a reduction in all-cause in-hospital mortality at 90 days in the group that received the CP. This result was unexpected considering the relatively small size of this study and the relatively low mortality rate. We recognize that in-hospital mortality data may not necessarily be representative of true mortality rates, but this is an important finding that needs further study and follow up. Interestingly, Sridhar et $\mathrm{al}^{12}$ found very similar results with their CP, noting no improvements in readmission rates but a significant reduction in deaths, supporting our finding.

\section{Limitations}

This study has some limitations. First, it is a retrospective study and therefore depended on data available in the electronic medical record system. As such, not all variables could be collected for every patient, preventing the inclusion of parameters such as $\mathrm{FEV}_{1}$, body mass index, pack-years of smoking history, and socioeconomic factors into the analysis. Second, the population had a particularly low average socioeconomic status and unusually high rates of SA and mental 
illness. The results of the study may therefore have reduced generalizability. Third, the care delivered to each patient was purposely unique and to assess specific interventions would need a much larger cohort.

\section{Conclusion}

We conclude that an individualized $\mathrm{CP}$ does not reduce readmissions in subjects admitted to hospital for AECOPD, but it does reduce readmissions in female patients and also decreases all-cause in-hospital mortality. Due to the individualized nature of our $\mathrm{CP}$, it is difficult to pinpoint the particular intervention(s) that lead to these effects. To tease out which aspects of the $\mathrm{CP}$ are more effective in improving patient outcomes, a larger randomized control trial that employs a similar model of care is needed to determine which specific interventions have the largest impact. We suspect that better and earlier recognition of exacerbations, improved self-management techniques, and availability of an Outreach Team for improving day-to-day disease management in the community could have contributed to the beneficial effects of the CP intervention following AECOPD.

\section{Acknowledgments}

The abstract of this paper was presented at the American Thoracic Society Conference 2015 in Denver, CO, USA, as a poster presentation with interim findings and was published in "Poster Abstracts" in the American Journal of Respiratory and Critical Care Medicine 191:2015:A1099. This research was supported by the British Columbia Lung Association. Dr van Eeden is the Canadian Institute for Health Research/ GlaxoSmithKline Professor in Chronic Obstructive Pulmonary Disease and Dr Sin is the Canada Research Chair in Chronic Obstructive Pulmonary Disease.

\section{Disclosure}

The authors report no conflicts of interest in this work.

\section{References}

1. Vestbo J, Hurd SS, Agusti AG, et al. Global strategy for the diagnosis, management, and prevention of chronic obstructive pulmonary disease: GOLD executive summary. Am J Respir Crit Care Med. 2013;187(4):347-365.

2. Suissa S, Dell'Aniello S, Ernst P. Long-term natural history of chronic obstructive pulmonary disease: severe exacerbations and mortality. Thorax. 2012;67(11):957-963.

3. Soler-Cataluna JJ, Martinez-Garcia MA, Roman Sanchez P, Salcedo E, Navarro M, Ochando R. Severe acute exacerbations and mortality in patients with chronic obstructive pulmonary disease. Thorax. 2005; 60(11):925-931.

4. Seemungal TA, Donaldson GC, Paul EA, Bestall JC, Jeffries DJ, Wedzicha JA. Effect of exacerbation on quality of life in patients with chronic obstructive pulmonary disease. Am J Respir Crit Care Med. 1998;157(5 Pt 1):1418-1422.
5. Makris D, Moschandreas J, Damianaki A, et al. Exacerbations and lung function decline in COPD: new insights in current and ex-smokers. Respir Med. 2007;101(6):1305-1312.

6. Mittmann N, Kuramoto L, Seung SJ, Haddon JM, Bradley-Kennedy C, Fitzgerald JM. The cost of moderate and severe COPD exacerbations to the Canadian healthcare system. Respir Med. 2008;102(3):413-421.

7. Discharge Abstract Database, Canadian Institute for Health Information, Fichier des hospitalisations MED-ÉCHO, ministère de la Santé et des Services sociaux du Québec. All-Cause Readmission to Acute Care and Return to the Emergency room. Ottawa, ON: Canadian Institute for Health Information; 2012.

8. Lemmens KM, Nieboer AP, Huijsman R. A systematic review of integrated use of disease-management interventions in asthma and COPD. Respir Med. 2009;103(5):670-691.

9. Titova E, Steinshamn S, Indredavik B, Henriksen AH. Long term effects of an integrated care intervention on hospital utilization in patients with severe COPD: a single centre controlled study. Respir Res. 2015;16:8.

10. Moullec G, Lavoie KL, Rabhi K, Julien M, Favreau H, Labrecque M. Effect of an integrated care programme on re-hospitalization of patients with chronic obstructive pulmonary disease. Respirology. 2012;17(4): 707-714.

11. Lawlor M, Kealy S, Agnew M, et al. Early discharge care with ongoing follow-up support may reduce hospital readmissions in COPD. Int $J$ Chron Obstruct Pulmon Dis. 2009;4:55-60.

12. Sridhar M, Taylor R, Dawson S, Roberts NJ, Partridge MR. A nurse led intermediate care package in patients who have been hospitalised with an acute exacerbation of chronic obstructive pulmonary disease. Thorax. 2008;63(3):194-200.

13. Monninkhof E, van der Valk P, van der Palen J, van Herwaarden C, Zielhuis G. Effects of a comprehensive self-management programme in patients with chronic obstructive pulmonary disease. Eur Respir J. 2003;22(5):815-820.

14. Jennings JH, Thavarajah K, Mendez M, Eichenhorn M, Kvale P, Yessayan L. Pre-discharge bundle for patients with acute exacerbations of chronic obstructive pulmonary disease to reduce readmissions and emergency room visits: a randomized, controlled trial. Chest. Epub 2014 Dec 24

15. American Thoracic Society; European Respiratory Society; 2004. Standards for the Diagnosis and Management of Patients with COPD. Available from: https://www.thoracic.org/copd-guidelines/resources/ copddoc.pdf. Accessed June 30, 2004.

16. McGhan R, Radcliff T, Fish R, Sutherland ER, Welsh C, Make B. Predictors of rehospitalization and death after a severe exacerbation of COPD. Chest. 2007;132(6):1748-1755.

17. Sharif R, Parekh TM, Pierson KS, Kuo YF, Sharma G. Predictors of early readmission among patients 40 to 64 years of age hospitalized for chronic obstructive pulmonary disease. Ann Am Thorac Soc. 2014; 11(5):685-694.

18. Filipowski M, Bozek A, Kozlowska R, Czyzewski D, Jarzab J. The influence of hospitalizations due to exacerbations or spontaneous pneumothoraxes on the quality of life, mental function and symptoms of depression and anxiety in patients with COPD or asthma. J Asthma. 2014;51(3):294-298.

19. Taylor SJ, Candy B, Bryar RM, et al. Effectiveness of innovations in nurse led chronic disease management for patients with chronic obstructive pulmonary disease: systematic review of evidence. BMJ. 2005; 331(7515):485.

20. Peytremann-Bridevaux I, Staeger P, Bridevaux PO, Ghali WA, Burnand B. Effectiveness of chronic obstructive pulmonary diseasemanagement programs: systematic review and meta-analysis. $A m J$ Med. 2008;121(5):433-443.e434.

21. Deans GD, Raffa JD, Lai C, et al. Mortality in a large community-based cohort of inner-city residents in Vancouver, Canada. CMAJ Open. 2013;1(2):E68-E76.

22. Linden IA, Mar MY, Werker GR, Jang K, Krausz M. Research on a vulnerable neighborhood-the Vancouver Downtown Eastside from 2001 to 2011. J Urban Health. 2013;90(3):559-573. 
23. Slaunwhite $\mathrm{AK}$. The role of gender and income in predicting barriers to mental health care in Canada. Community Ment Health J. 2015;51(5):621-627.

24. Bos-Touwen I, Schuurmans M, Monninkhof EM, et al. Patient and disease characteristics associated with activation for self-management in patients with diabetes, chronic obstructive pulmonary disease, chronic heart failure and chronic renal disease: a cross-sectional survey study. PLoS One. 2015;10(5):e0126400.

25. Hayton C, Clark A, Olive $\mathrm{S}$, et al. Barriers to pulmonary rehabilitation: characteristics that predict patient attendance and adherence. Respir Med. 2013;107(3):401-407.

26. Drummond MB, Merlo CA, Astemborski J, et al. The effect of HIV infection on longitudinal lung function decline among IDUs: a prospective cohort. AIDS. 2013;27(8):1303-1311.

27. Camp PG, Coxson HO, Levy RD, et al. Sex differences in emphysema and airway disease in smokers. Chest. 2009;136(6):1480-1488.
28. Martinez FJ, Curtis JL, Sciurba F, et al. Sex differences in severe pulmonary emphysema. Am J Respir Crit Care Med. 2007;176(3):243-252.

29. Kilic H, Kokturk N, Sari G, Cakir M. Do females behave differently in COPD exacerbation? Int J Chron Obstruct Pulmon Dis. 2015;10: $823-830$.

30. Katsura H, Yamada K, Wakabayashi R, Kida K. Gender-associated differences in dyspnoea and health-related quality of life in patients with chronic obstructive pulmonary disease. Respirology. 2007;12(3): 427-432.

31. Di Marco F, Verga M, Reggente M, et al. Anxiety and depression in COPD patients: the roles of gender and disease severity. Respir Med. 2006; 100(10):1767-1774 


\section{Supplementary materials}

Table SI Summary of effect of CP on readmissions in whole population

\begin{tabular}{lllll}
\hline Readmission & \multicolumn{2}{l}{ Average number of readmissions per patient } & Adjusted P-value & Unadjusted $P$-value \\
\cline { 2 - 4 } & Pre-CP & CP & \\
\hline T30 & 0.4059 & 0.3613 & 0.62147 & 0.562 \\
ER30 & 0.1956 & 0.1571 & 0.44038 & 0.481 \\
AC30 & 0.2103 & 0.2042 & 0.9329 & 0.894 \\
T90 & 0.9557 & 0.7592 & 0.124529 & 0.146 \\
ER90 & 0.5055 & 0.3560 & 0.13307 & 0.162 \\
AC90 & 0.487 1 & 0.4031 & 0.26266 & 0.246 \\
\hline
\end{tabular}

Notes: Summary of readmission rates in the pre-CP and CP groups. P-values are shown adjusted for covariates (age, sex, number of comorbidities, index admission length of stay, substance abuse, and mental illness) and not adjusted.

Abbreviations: T30, total readmissions in 30 days; ER30, emergency room readmissions in 30 days; AC30, acute care readmissions in 30 days; T90, total readmissions in 90 days; ER90, emergency room readmissions in 90 days; AC90, acute care readmissions in 90 days; CP, care package.

Table S2 Summary of effect of SA on readmissions

\begin{tabular}{lllll}
\hline Readmission & \multicolumn{2}{l}{ Average number of readmissions per patient } & Adjusted $\boldsymbol{P}$-value & Unadjusted $\boldsymbol{P}$-value \\
\cline { 2 - 4 } & SA & NSA & & \\
\hline T30 & 0.7349 & 0.3113 & 0.00189 & $1.68 \mathrm{e}-05$ \\
ER30 & 0.4217 & 0.1266 & 0.0108 & $4.27 \mathrm{e}-06$ \\
AC30 & 0.3133 & 0.1847 & 0.0317 & 0.0847 \\
T90 & 1.614 & 0.7124 & 0.000302 & $3.66 \mathrm{e}-08$ \\
ER90 & 0.9518 & 0.3325 & 0.01098 & $1.10 \mathrm{e}-06$ \\
AC90 & 0.6627 & 0.4063 & 0.0275 & 0.00688 \\
\hline
\end{tabular}

Notes: Summary of readmission rates in patients with SA and without NSA comorbid substance abuse. P-values are shown adjusted for covariates (age, sex, care package, number of comorbidities, index admission length of stay, and mental illness) and not adjusted.

Abbreviations: SA, substance abuse; NSA, nonsubstance abuse; T30, total readmissions in 30 days; ER30, emergency room readmissions in 30 days; AC30, acute care readmissions in 30 days; T90, total readmissions in 90 days; ER90, emergency room readmissions in 90 days; AC90, acute care readmissions in 90 days.

Table S3 Summary of effect of CP on readmissions depending on presence of SA

\begin{tabular}{|c|c|c|c|c|c|c|c|c|}
\hline \multirow[t]{3}{*}{ Readmission } & \multicolumn{4}{|l|}{ SA } & \multicolumn{4}{|l|}{ NSA } \\
\hline & \multicolumn{2}{|c|}{$\begin{array}{l}\text { Average number of } \\
\text { readmissions per patient }\end{array}$} & \multirow[t]{2}{*}{$\begin{array}{l}\text { Adjusted } \\
P \text {-value }\end{array}$} & \multirow[t]{2}{*}{$\begin{array}{l}\text { Unadjusted } \\
\text { P-value }\end{array}$} & \multicolumn{2}{|c|}{$\begin{array}{l}\text { Average number of } \\
\text { readmissions per patient }\end{array}$} & \multirow[t]{2}{*}{$\begin{array}{l}\text { Adjusted } \\
P \text {-value }\end{array}$} & \multirow[t]{2}{*}{$\begin{array}{l}\text { Unadjusted } \\
P \text {-value }\end{array}$} \\
\hline & Pre-CP & CP & & & Pre-CP & CP & & \\
\hline T30 & 0.7200 & 0.7576 & 0.913 & 0.974 & 0.3348 & 0.2785 & 0.4450 & 0.523 \\
\hline ER30 & 0.4200 & 0.4242 & 0.855 & 0.786782 & 0.1448 & 0.1013 & 0.33372 & $0.54 I$ \\
\hline AC30 & 0.3000 & 0.3333 & 0.6194 & 0.729314 & 0.1900 & 0.1772 & 0.888 & 0.735 \\
\hline T90 & 1.700 & 1.485 & 0.543 & 0.457 & 0.7873 & 0.6076 & 0.12757 & 0.237 \\
\hline ER90 & 0.9600 & 0.9394 & 0.791 & 0.643 & 0.4027 & 0.2342 & 0.081980 & 0.189 \\
\hline $\mathrm{AC} 90$ & 0.7400 & 0.5455 & 0.404 & 0.412 & 0.4299 & 0.3734 & 0.4325 & 0.436 \\
\hline
\end{tabular}

Notes: Summary of readmission rates in pre-CP and CP NSA and SA patients. P-values are shown adjusted for covariates (age, sex, care package, number of comorbidities, index admission length of stay, and mental illness) and not adjusted.

Abbreviations: SA, substance abuse; NSA, nonsubstance abuse; CP, care package; T30, total readmissions in 30 days; ER30, emergency room readmissions in 30 days; $\mathrm{AC} 30$, acute care readmissions in 30 days; T90, total readmissions in 90 days; ER90, emergency room readmissions in 90 days; AC90, acute care readmissions in 90 days. 
Table S4 Summary of effect of sex on readmissions

\begin{tabular}{lllll}
\hline Readmission & \multicolumn{2}{l}{ Average number of readmissions per patient } & Adjusted $P$-value & Unadjusted P-value \\
\cline { 2 - 4 } & Females & Males & \\
\hline T30 & 0.3257 & 0.4251 & 0.20001 & 0.204 \\
ER30 & 0.1829 & 0.1777 & 0.9528 & 0.926 \\
AC30 & 0.1429 & 0.2474 & 0.0210 & 0.025545 \\
T90 & 0.8629 & 0.8815 & 0.781237 & 0.892 \\
ER90 & 0.4171 & 0.4599 & 0.67975 & 0.694 \\
AC90 & 0.4457 & 0.4564 & 0.7397 & 0.884 \\
\hline
\end{tabular}

Notes: Summary of readmission rates in male and female patients. P-values are shown adjusted for covariates (age, care package, number of comorbidities, index admission length of stay, substance abuse, and mental illness) and not adjusted.

Abbreviations: T30, total readmissions in 30 days; ER30, emergency room readmissions in 30 days; AC30, acute care readmissions in 30 days; T90, total readmissions in 90 days; ER90, emergency room readmissions in 90 days; AC90, acute care readmissions in 90 days.

Table S5 Summary of effect of CP on readmissions depending on sex

\begin{tabular}{|c|c|c|c|c|c|c|c|c|}
\hline \multirow[t]{3}{*}{ Readmission } & \multicolumn{4}{|l|}{ Females } & \multicolumn{4}{|l|}{ Males } \\
\hline & \multicolumn{2}{|c|}{$\begin{array}{l}\text { Average number of } \\
\text { readmissions per patient }\end{array}$} & \multirow[t]{2}{*}{$\begin{array}{l}\text { Adjusted } \\
P \text {-value }\end{array}$} & \multirow[t]{2}{*}{$\begin{array}{l}\text { Unadjusted } \\
P \text {-value }\end{array}$} & \multicolumn{2}{|c|}{$\begin{array}{l}\text { Average number of } \\
\text { readmissions per patient }\end{array}$} & \multirow[t]{2}{*}{$\begin{array}{l}\text { Adjusted } \\
P \text {-value }\end{array}$} & \multirow[t]{2}{*}{$\begin{array}{l}\text { Unadjusted } \\
P \text {-value }\end{array}$} \\
\hline & Pre-CP & $\mathbf{C P}$ & & & Pre-CP & CP & & \\
\hline T30 & 0.3750 & 0.2658 & 0.3672 & 0.315 & 0.4229 & 0.4286 & 0.9879 & 0.957 \\
\hline ER30 & 0.2396 & 0.1139 & 0.17592 & 0.153 & 0.1714 & 0.1875 & $0.9797 \mid$ & 0.819084 \\
\hline $\mathrm{AC} 30$ & 0.1354 & 0.1519 & 0.711 & 0.758163 & 0.2514 & 0.2411 & 0.9604 & 0.877 \\
\hline T90 & 1.073 & 0.6076 & 0.0205 & 0.0174 & 0.8914 & 0.8661 & 0.721703 & 0.89 \\
\hline ER90 & 0.6042 & 0.1899 & 0.00414 & 0.00376 & 0.4514 & 0.4732 & 0.936407 & 0.884 \\
\hline AC90 & 0.4688 & 0.4177 & 0.690 & 0.654 & $0.497 \mid$ & 0.3929 & $0.2344 I$ & 0.269 \\
\hline
\end{tabular}

Notes: Summary of readmission rates in pre-CP and CP male and female patients. P-values are shown adjusted for covariates (age, number of comorbidities, index admission length of stay, substance abuse, and mental illness) and not adjusted.

Abbreviations: CP, care package; T30, total readmissions in 30 days; ER30, emergency room readmissions in 30 days; AC30, acute care readmissions in 30 days; T90, total readmissions in 90 days; ER90, emergency room readmissions in 90 days; AC90, acute care readmissions in 90 days.

\section{Publish your work in this journal}

The International Journal of COPD is an international, peer-reviewed journal of therapeutics and pharmacology focusing on concise rapid reporting of clinical studies and reviews in COPD. Special focus is given to the pathophysiological processes underlying the disease, intervention programs, patient focused education, and self management protocols.

\section{Dovepress}

This journal is indexed on PubMed Central, MedLine and CAS. The manuscript management system is completely online and includes a very quick and fair peer-review system, which is all easy to use. Visit $\mathrm{http}: / / \mathrm{www}$. dovepress.com/testimonials.php to read real quotes from published authors. 